Research Paper

\title{
Ethanol Extract from Brucea Javanica Seed Inhibits Angiogenesis Mediated by Platelet Derived Growth Factor Receptor-beta
}

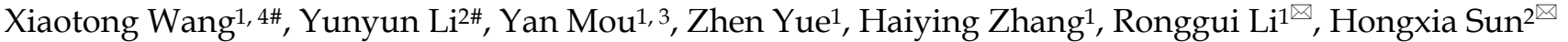 \\ 1. Key Laboratory of Pathobiology, Ministry of Education, Norman Bethune College of Medicine, Jilin University, Changchun, China \\ 2. People's Hospital of Jilin Province and Changchun University of Chinese Medicine, Changchun, P.R. China \\ 3. Second Hospital of Jilin University, Changchun, China \\ 4. Current address: Institute of Hematology and Blood Diseases Hospital, Chinese Academy of Medical Sciences, Tianjin, China \\ \#These authors contributed equally to this work. \\ $\triangle$ Corresponding authors: Dr. Ronggui Li, The Key Laboratory of Pathobiology, Ministry of Education, Norman Bethune College of Medicine, Jilin University, \\ Changchun, 130021, P.R. China. Tel.: 86-431 85619481; E-mail: lirg@jlu.edu.cn and Dr. Hongxia Sun, People's Hospital of Jilin Province and Changchun \\ University of Chinese Medicine, Changchun, P.R. China. Tel.: 86-431 85595280; E-mail:984897342@qq.com. \\ (C) Ivyspring International Publisher. This is an open access article distributed under the terms of the Creative Commons Attribution (CC BY-NC) license \\ (https://creativecommons.org/licenses/by-nc/4.0/). See http://ivyspring.com/terms for full terms and conditions.
}

Received: 2018.07.05; Accepted: 2018.08.29; Published: 2018.10.20

\begin{abstract}
The present study aimed to investigate the effects of ethanol extract from Brucea javanicaseed (EEBJS) on the angiogenesis of human umbilical vein endothelial cells (HUVECs) and the possible molecular signal involved. Firstly, a Matrigel-based in vitro angiogenesis assay demonstrated that EEBJS inhibited the angiogenesis of HUVECs in a dose-dependent manner. Then by using porcine aortic endothelial cells which stably express human PDGFR-beta, we found that the inhibition of angiogenesis was mediated by PDGFR-beta. Taken together, we conclude that EEBJS inhibited the angiogenesis function of the vascular endothelial cells mediated by PDGFR-beta, and postulate that it might contribute to the therapeutic effects of EEBJS on malignant tumors.
\end{abstract}

Key words: Ethanol Extract from Brucea javanica Seed; Angiogenesis; Human Umbilical Vein Endothelial Cells; Platelet Derived Growth Factor Receptor; Malignant Tumors

\section{Introduction}

Brucea javanica is an evergreen shrub distributed widely in Southeast Asia and northern Australia [1]. The seed of Brucea javanica, as a traditional Chinese herbal medicine, has been broadly used for treatment of various cancers $[1,2]$. Over the past several decades, its anticancer properties have been examined in a large number of studies $[3,4]$. Several active substances have been identified and studies of the molecular mechanisms of its anticancer effect are ongoing [2, 5]. However, thus far, no single compound extracted from this herb has been used in clinical treatment of cancer, and its anticancer mechanisms are little known. Therefore, its application has been confined to alternative treatments [2].

One of the important 'hallmarks' of cancer is angiogenesis, which is the process of formation of new blood vessels that are necessary for tumor expansion, invasion and metastasis.[6, 7]. Angiogenesis is a major function of vascular endothelial cells [8]. In cancers, angiogenesis is largely mediated by alterations of receptor tyrosine kinase pathways [9-11]. In a previous study, we found that platelet derived growth factor receptor-beta (PDGFR-beta), plays an important role in mediating the angiogenesis of HUVECs [12]. It has also been reported that PDGF signaling is involved in angiogenesis of malignant stroma to support the proliferation of breast cancer cell [13]. However, studies about the effects of EEBJS on the angiogenesis of HUVECs have not been reported, nor have potential mechanisms been elucidated. Therefore, in the present study, we 
analyzed the effects of EEBJS on the angiogenesis of HUVECs and examined a possible role of PDGFR-beta, in order to identify a molecular signal through which EEBJS might inhibit tumor growth.

\section{Materials and Methods}

\section{Materials}

Human Umbilical Vein Endothelial Cells (HUVECs) and endothelial cell medium (ECM,) were purchased from the ScienCell Research Laboratories (San Diego, USA). Porcine aortic endothelial cells with stably transfected human PDGFR-beta were from Professor Rainer Heuchel, Karolinska Institute, Sweden. IMDM was purchased from Gibco BRL (Rockville, USA). Fetal bovine serum was purchased from HyClone Inc. (Logan, USA). Endothelial cell growth supplement (ECGS) was purchased from ScienCell Research Laboratories (San Diego, USA). The In Vitro Angiogenesis Assay Kit was purchased from Millipore (Billerica, USA). Calcein-AM was purchased from Santa Cruz Biotechnology, Inc. (Dallas, USA). Brucea javanica seed was purchased from Changchun pharmacy (Changchun, China). Ethanol extract of Brucea javanica seed were prepared in the Key Laboratory of Pathobiology, Ministry of Education (Changchun, China).

\section{Cell culture and treatments}

The HUVECs were grown in ECM medium containing $5 \%$ FBS and $1 \%$ endothelial cell growth supplement (ECGS). PDGFR-beta/PAE cells were grown in IMDM containing $10 \%$ FBS. Both cell types were incubated at $37^{\circ} \mathrm{C}$ in $5 \% \mathrm{CO}_{2}$ and a humidified atmosphere. HUVECs were used for all experiments at passages 2 to 6 . For EEBJS treatment, the cells were plated in $6 \mathrm{~cm}$ diameter dishes at a density of $0.5 \times 10^{5}$ cells per dish. After incubating them for 24 hours, the medium was exchanged with fresh medium containing various concentrations of EEBJS or vehicle, as indicated in Figures 1 and 2, and incubated for another 24 hours.

\section{In vitro angiogenesis assay}

The angiogenesis of the cells was evaluated by a Matrigel in vitro angiogenesis assay technique. The assay was performed with a detailed procedure as described previously [14]. Briefly, $100 \mu \mathrm{l}$ stock solution of Matrigel was added to each well in 48-well plates and kept at $37^{\circ} \mathrm{C}$ for $30 \mathrm{~min}$ in order to form the Matrigel. Cell suspensions containing $3 \times 10^{4}$ cells in $100 \mu \mathrm{l}$ of ECM were seeded on the Matrigel of each well, and incubated for 6 hours. Then Calcein-AM (0.1 $\mathrm{mM}$ ) was directly added to each well for $20 \mathrm{~min}$ at $37^{\circ} \mathrm{C}$ to stain the cells which were imaged under a phase contrast microscope with an excitation wavelength of $490 \mathrm{~nm}$ and an emission wavelength of $515 \mathrm{~nm}$. For quantification, the values for the pattern recognition, branch point and total capillary tube length were determined following the manufacturer's guidelines (ECM625; Millipore). Image J software was used in the first instance prior to double-checking by an independent assessor. 5 random microscopic $(\times 100)$ fields per well were included and the data are expressed as mean \pm SD of 5 samples.

\section{Statistical analysis}

All calculations and statistical analyses were performed by using GraphPad Prism 5.0 software (San Diego, USA). T test was used to analyze the significance of any differences between two groups. The statistical significance was defined as $p<0.05$.

\section{Results}

\section{EEBJS inhibited angiogenesis of HUVECs in a dose-dependent manner}

To evaluate the effects of EEBJS on the angiogenesis of HUVECs, an in vitro angiogenesis assay was performed for the cells exposed to varying concentration of EEBJS. Fig. 1A shows representative microscopic appearances. Cells not exposed to EEBJS displayed morphologic features of angiogenesis, specifically, cells aligned themselves; there was formation of capillary tubes with or without sprouting; there was formation of closed polygons and/or complex mesh-like structures. Upon exposure to EEJBF, incomplete network formation and fewer branch points or tubular structures were found. In the cells exposed to higher concentration of EEBJS (10ìg/ml and 100ìg/ml in Fig. 1A) the morphologic appearances for angiogenesis almost completely disappeared. Fig. 1B, 1C and 1D show the dose dependent effects on parameters of pattern recognition, branch points and total tube lengths, each reflecting a different aspect of angiogenesis. 1D shows the IC50 values for these parameters. Clearly, EEBJS inhibited angiogenesis of HUVECs in dose dependent manner.

\section{EEBJS inhibited the angiogenesis mediated by PDGFR-beta}

Our previous study showed that platelet derived growth factor receptor-beta (PDGFR-beta), plays an important role in mediating angiogenesis of HUVECs [12]. To determine whether EEBJS inhibits angiogenesis, mediated by PDGFR-beta, we utilized porcine aortic endothelial cells with stably transfected human PDGF beta-receptors (PDGFR-beta/PAE cells) which have been previously employed to examine PDGF-BB triggered cytoplasmic calcium responses [15] and proved that only PDGFR-beta is responsible for the 
angiogenesis of the cells [12]. As shown in Fig. 2A, the cells unexposed to EEBJS presented uniform networks with dense branch points and intensive tube structures $(0 \mathrm{ig} / \mathrm{ml}$ in Fig. 2A). Compared with the unexposed cells, less typical morphologic appearances for angiogenesis were observed in the cells exposed to EEJBF. In the cells exposed to higher concentration of EEBJS (100ig/ml in Fig. 2A) the morphologic appearances for angiogenesis completely disappeared. Figure 2B, 2C and 2D are statistically analyzed results which show the dose dependent effects on parameters of pattern recognition, branch points and total tube lengths upon EEBJS treatment, each reflecting a different aspect of angiogenesis. 2D shows the IC50 values for these parameters. It showed clearly that EEBJS inhibited the angiogenesis of the cells in a dose dependent manner. These data support the conclusion that EEBJS inhibits the angiogenesis function of the vascular endothelial cells, mediated by PDGFR-beta. We postulate this effect might play an important role in the therapeutic effect of EEBJS on malignant tumors.

A
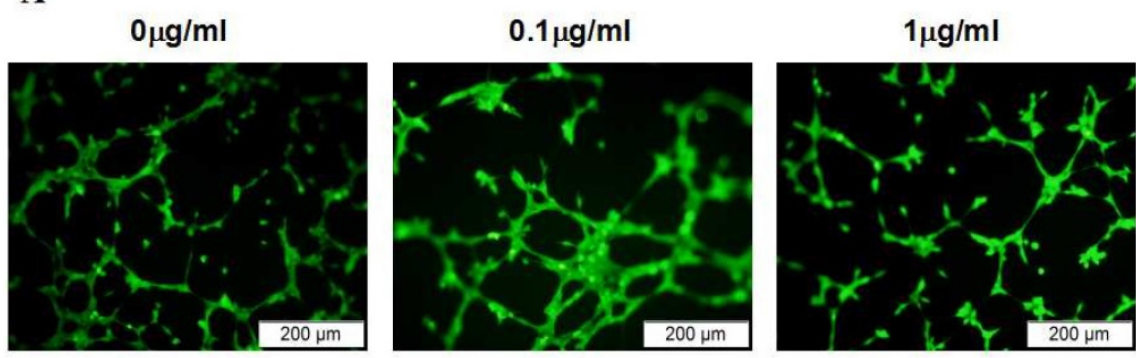

$10 \mu \mathrm{g} / \mathrm{ml}$ $100 \mu \mathrm{g} / \mathrm{ml}$
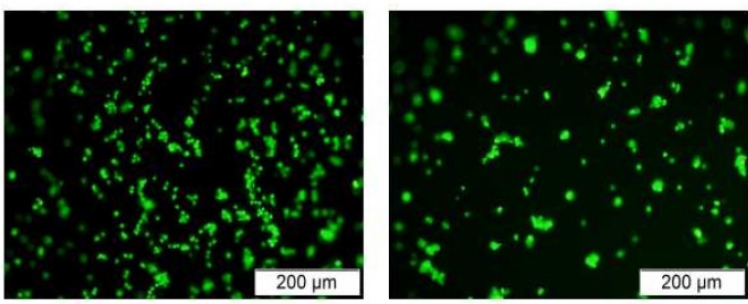

B
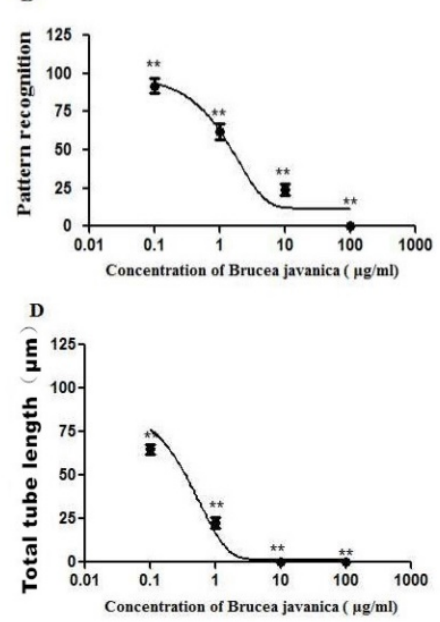

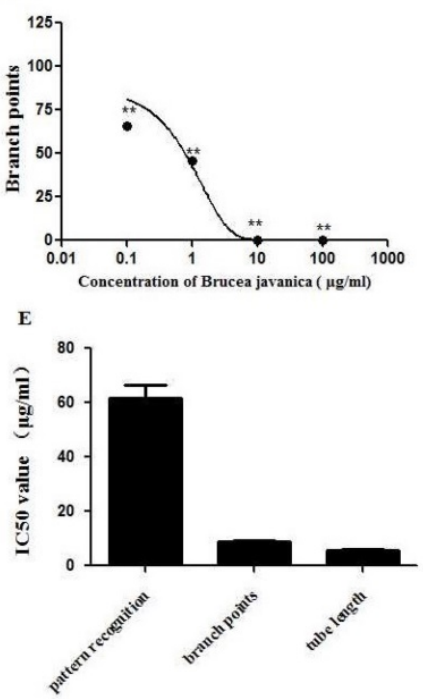

Figure 1. EEBJS suppressed the angiogenesis of HUVECs in dose-dependent manner. HUVECs were exposed to various concentrations of EEBJS, as indicated. The angiogenesis assay, cell staining and the values quantification for the pattern recognition, branch point and total capillary tube length are described in the Methods section. Representative microscopic fields are shown (A). Dose-dependent decreases of angiogenesis were plotted by using a nonlinear regression model $(B, C$ and $D)$ and the data are expressed relative to that of the control cells without exposure to EEBJS. The data are expressed as the mean $\pm S D$. $N=5$, and **P $<0.01$ versus the control cells without exposure to EEBJS. IC50 values (E) were determined based on the fitted curves. 
$\mathbf{A}$
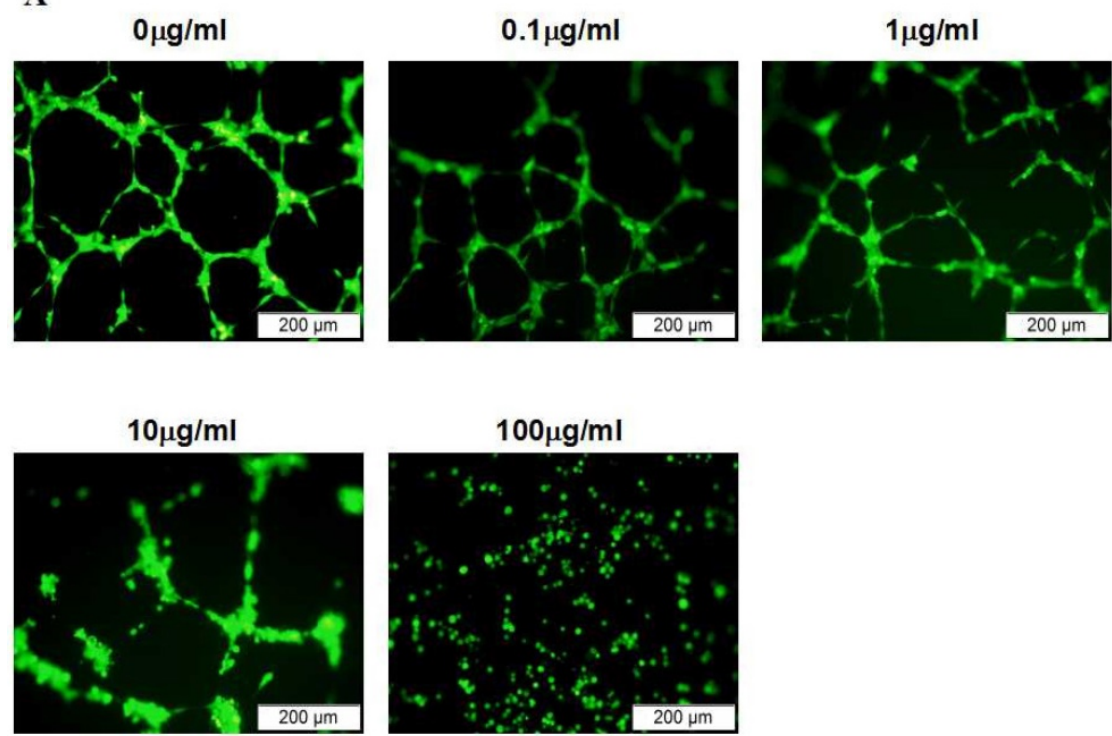

F
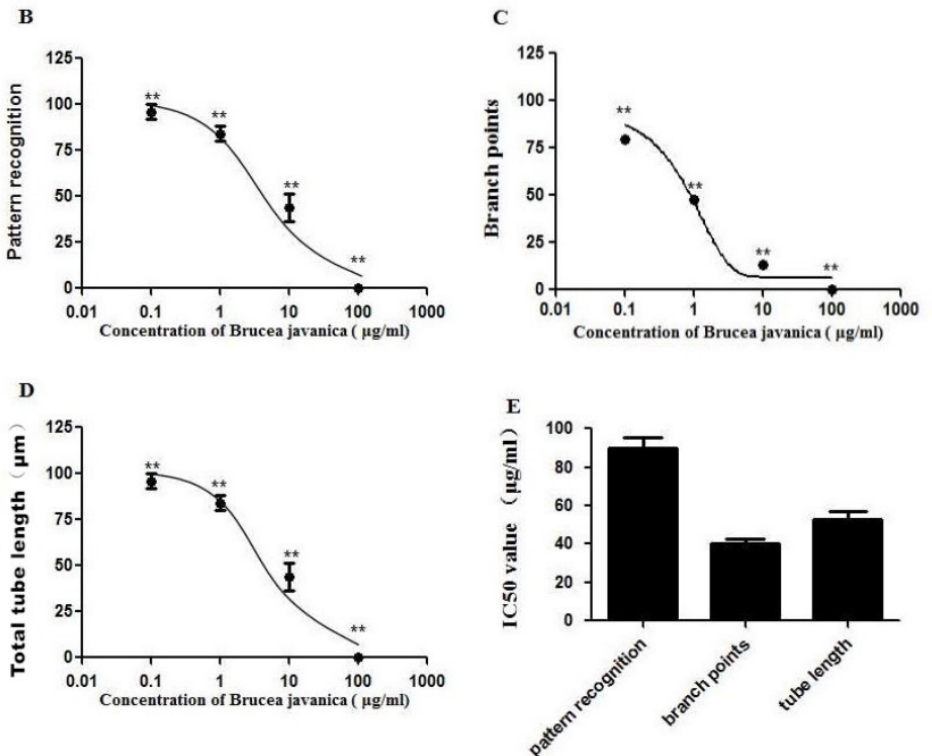

Figure 2. Dose-dependent suppression of the angiogenesis of PDGFR-beta/PAE cells after exposure to EEBJS. PDGFR-beta/PAE cells were exposed to various concentrations of EEBJS, as indicated. The angiogenesis assay and the values quantification for the pattern recognition, branch point and total capillary tube length were performed as described in method section. Representative microscopic fields are shown in A. Dose-dependent decreases of angiogenesis were plotted by using a nonlinear regression model $(\mathrm{B}, \mathrm{C}$ and $\mathrm{D})$. The data are expressed relative to that of the control cells without exposure to $\mathrm{EEB}$ JS. $\mathrm{N}=5$, and $* * \mathrm{P}<0.01$ versus the control cells without exposure to EEBJS. IC50 values $(E)$ were determined based on the fitted curves.

\section{Discussion}

In the present study, we found that EEBJS suppressed the angiogenesis of HUVECs in a dose-dependent manner, suggesting that inhibition of the angiogenesis of the vascular endothelial cells might contribute to a therapeutic effect of EEBJS on malignant tumors. This conclusion was supported by the studies that malignant stroma increases luminal breast cancer cell proliferation and angiogenesis [13].

Platelet-derived growth factor (PDGF) is believed to be an important mitogen for connective tissue, having roles during embryonic development [16]. It has been reported that the PDGF signaling pathways contributes to the development of a vascular system which involves the assembly of endothelial cells and vascular smooth muscle cells/pericytes (vSMC/PC) into many different types of blood vessels [17]. Hellstrom et al. found that PDGFBB, secreted by endothelial cells, recruits PDGFR-beta positive vSMC/PC cells and stimulates their proliferation at sites of endothelial PDGF-B expression, in the mouse [17]. In the present study, by 
using porcine aortic endothelial cells with stably transfected human PDGFR-beta, we found that EEBJS suppressed the angiogenesis mediated by PDGFR-beta of vascular endothelial cells. This conclusion was also supported by our earlier study in which PDGFR-beta has been proved play a crucial role in regulating angiogenesis of human vascular endothelial cells by application of PDGFR-betaselective inhibitor, CP-673451 [12].

Taken together, our results provide evidence to support that EEBJS inhibited the angiogenesis of vascular endothelial cells mediated by PDGFR-beta, and postulate that it might contribute to the therapeutic effects of EEBJS on malignant tumors because PDGF overexpression has been linked to different types of malignancies and particularly, it has been reported that PDGF produced by malignant stromal cells pays an important role in the enhancesment of cancer cell proliferation and angiogenesis [13]. Present study provides additive knowledge on how EEBJS affect PDGF and its receptor which might be used in developing antiangiogenic strategies for the treatment of malignant disease.

\section{Conclusions}

EEBJS suppressed the angiogenesis of HUVECs in a dose-dependent manner. PDGFR-beta plays a crucial role in mediating this angiogenesis. Taken together, we conclude that EEBJS inhibited the angiogenesis function of the vascular endothelial cells, mediated by PDGFR-beta, and postulate that PDGFR-beta may contribute to the therapeutic effects of EEBJS on malignant tumors.

\section{Abbreviations}

ECGS: endothelial cell growth supplement; ECM: endothelial cell medium; EEBJS: ethanol extract from Brucea javanicaseed; HUVECs: human umbilical vein endothelial cells; IC50: half maximal inhibitory concentration; PAE: porcine aortic endothelial cells; PDGF-B: platelet Derived Growth Factor chain B; PDGFR-beta: platelet Derived Growth Factor Receptor-beta; vSMC/PC: vascular smooth muscle cells/pericytes.

\section{Acknowledgments}

This study was supported by the National Natural Science Foundation of China (Grants: NSFC No. 21277057). We would like to express our great appreciation to Professor F. William Orr from the University of Manitoba in Canada for his help in revising the manuscript.

\section{Competing Interests}

The authors have declared that no competing interest exists.

\section{References}

1. Zhao L, Li C, Zhang Y, Wen Q, Ren D. Phytochemical and biological activities of an anticancer plant medicine: Brucea javanica. Anticancer Agents Med Chem. 2014; 14: 440-58.

2. Yan Z, Guo GF, Zhang B. Research of Brucea javanica against cancer. Chin J Integr Med. 2017; 23: 153-60.

3. Nie YL, Liu KX, Mao XY, Li YL, Li J, Zhang MM. Effect of injection of brucea javanica oil emulsion plus chemoradiotherapy for lung cancer: a review of clinical evidence. J Evid Based Med. 2012; 5: 216-25.

4. Xu W, Jiang X, Xu Z, Ye T, Shi Q. The Efficacy of Brucea javanica Oil Emulsion Injection as Adjunctive Therapy for Advanced Non-Small-Cell Lung Cancer: A Meta-Analysis. Evid Based Complement Alternat Med. 2016; 2016: 5928562.

5. Chen M, Chen R, Wang S, Tan W, Hu Y, Peng X, et al. Chemical components, pharmacological properties, and nanoparticulate delivery systems of Brucea javanica. Int J Nanomedicine. 2013; 8: 85-92.

6. Amini A, Masoumi Moghaddam S, Morris DL, Pourgholami MH. The critical role of vascular endothelial growth factor in tumor angiogenesis. Curr Cancer Drug Targets. 2012; 12: 23-43.

7. Hu Z, Brooks SA, Dormoy V, Hsu CW, Hsu HY, Lin LT, et al. Assessing the carcinogenic potential of low-dose exposures to chemical mixtures in the environment: focus on the cancer hallmark of tumor angiogenesis. Carcinogenesis. 2015; 36 Suppl 1: S184-202.

8. Hofer E, Schweighofer B. Signal transduction induced in endothelial cells by growth factor receptors involved in angiogenesis. Thromb Haemost. 2007; 97: 355-63.

9. Zhang X, Simons M. Receptor tyrosine kinases endocytosis in endothelium: biology and signaling. Arterioscler Thromb Vasc Biol. 2014; 34: 1831-7.

10. Jeltsch M, Leppanen VM, Saharinen P, Alitalo K. Receptor tyrosine kinase-mediated angiogenesis. Cold Spring Harb Perspect Biol. 2013; 5.

11. Tohyama O, Matsui J, Kodama K, Hata-Sugi N, Kimura T, Okamoto K, et al. Antitumor activity of lenvatinib (e7080): an angiogenesis inhibitor that targets multiple receptor tyrosine kinases in preclinical human thyroid cancer models. J Thyroid Res. 2014; 2014: 638747.

12. Wang X, Mou Y, Yue Z, Zhang H, Su X, Wang Y, et al. Arsenite suppresses angiogenesis of vascular endothelial cells mediated by Platelet Derived Growth Factor Receptor-beta. Environ Toxicol Pharmacol. 2016; 46: 168-73.

13. Pinto MP, Dye WW, Jacobsen BM, Horwitz KB. Malignant stroma increases luminal breast cancer cell proliferation and angiogenesis through platelet-derived growth factor signaling. BMC Cancer. 2014; 14.

14. Mou Y, Yue Z, Wang X, Li W, Zhang H, Wang Y, et al. OCT4 Remodels the Phenotype and Promotes Angiogenesis of HUVECs by Changing the Gene Expression Profile. Int J Med Sci. 2016; 13: 386-94.

15. Ridefelt P, Yokote K, Claesson-Welsh L, Siegbahn A. PDGF-BB triggered cytoplasmic calcium responses in cells with endogenous or stably transfected PDGF beta-receptors. Growth Factors. 1995; 12: 191-201.

16. Appelmann I, Liersch R, Kessler T, Mesters RM, Berdel WE. Angiogenesis inhibition in cancer therapy: platelet-derived growth factor (PDGF) and vascular endothelial growth factor (VEGF) and their receptors: biological functions and role in malignancy. Recent Results Cancer Res. 2010; 180: 51-81.

17. Hellstrom M, Kalen M, Lindahl P, Abramsson A, Betsholtz C. Role of PDGF-B and PDGFR-beta in recruitment of vascular smooth muscle cells and pericytes during embryonic blood vessel formation in the mouse. Development. 1999; 126: 3047-55. 